\title{
Original Afrticle \\ Morbidity and Mortality Pattern of Neonates Admitted into Nursery Unit in Wad Medani Hospital, Sudan \\ Elhassan M. Elhassan ${ }^{1}$, Ahmed A. Hassanb ${ }^{1}$, Omer A. Mirghani ${ }^{1}$, Ishag Adam ${ }^{2 *}$
}

\begin{abstract}
:
Background: Neonatal outcome is an important indicator of obstetrics and health care. Few or no published data are available concerning neonatal morbidity and mortality in Sudan.

Objectives: To study morbidity and mortality pattern amongst inborn neonates admitted into nursery unit in Wad Medani Hospital, Sudan during the period Jan-June, 2009.

Results: A total of 1211 (29.5\%) out of 4098 in-born neonates were admitted during study period. The major indications for neonatal admission were; infections $300(24.8 \%)$, low birth weight (LBW) 307(25.4\%) and asphyxia 130(10.7). There were 133(11\%) neonatal deaths; preterm delivery, LBW and birth asphyxia were the major causes of death among these neonates.

Conclusion: neonatal infection, preterm birth and LBW were the common causes of neonatal morbidity and mortality. There is an urgent need for more research throughout the country concerning these common causes of morbidity and mortality.
\end{abstract}

Key words: pregnancy, preterm delivery, low birth weight, asphyxia.

$\mathrm{N}$ eonatal outcome is an important indicator of obstetrics and health care. About four million infants die during the first month of life worldwide ${ }^{1}$. The vast majority of these neonatal deaths occur in poor countries where standards of neonatal care are low. One of the Millennium Development Goals is to reduce the number of childhood deaths under the age of five years by two thirds by $2015^{2}$. To achieve this goal, a substantial reduction in early neonatal deaths will be required. The first steps in improving early neonatal survival are to document the number and rate of deaths, and identify their common causes. Few or no published data are available concerning neonatal morbidity and mortality in Sudan, which is the largest African country- area wise- with 40 million inhabitants. Thus, the current study was conducted to provide caregivers and health planners with basic data necessary for interventions to reduce neonatal morbidity and mortality.

1.University of Geizera, Wad Medani, Sudan.

2. University of Khartoum, Sudan

* Correspondence: TeL+249912168988

Fax +249183771211

E-Mail:ishagadam@hotmail.com
This will add to previous research on maternal, perinatal and neonatal epidemiology $\mathrm{y}^{3-8}$.

\section{Materials and methods}

The morbidity and mortality pattern amongst inborn neonates admitted into nursery unit in Wad Medani Maternity Hospital, Sudan during the period January-June, 2009 were reviewed. This hospital is the only governmental hospital that provides maternity services in the city, in addition to high percentage of referral of high risk pregnancies from other peripheral hospitals. The medical files of the neonates were retrospectively reviewed. The data collected included maternal age, maternal gestational age, mode of deliveries, babies sex, birth weight, cause of admission (diagnosis), duration of stay, and final outcome of these babies (death, discharge, discharged against medical advice).

\section{Definitions}

Preterm was defined as live born neonate delivered before 37 weeks from 1st day of last menstrual period and confirmed clinically following the delivery ${ }^{9}$, Low birth weight (LBW) was define as birth weight $<2500 \mathrm{~g}$. Neonatal infections (sepsis, pneumonia) were 
diagnosed on clinical ground along with positive blood culture examination if required. Meconium aspiration syndrome was diagnosed both radiologically and clinically based on; born with meconium-stained amniotic fluid, chest radiograph and had respiratory distress that persisted beyond 24 hours and required oxygen. Congenital heart malformations were diagnosed based on clinical features and/or using other facilities i.e. ultrasound, ECG changes, Chest X-Ray, and echocardiography. Birth asphyxia was mainly clinically diagnosed (APGAR score of $<7$ at $5 \mathrm{~min})$. Neonatal jaundice was diagnosed clinically, and by assessing of serum bilirubin.

Statistics: Data were entered in computer using SPSS for windows and analyzed. Means and percentages were calculated.

Ethics: The study received ethical clearance from the Board at Wad Medani Hospital

\section{Results:}

A total of 1211 (29.5\%) out of 4098 in-born neonates were admitted into nursery unit for a mean (SD) of 4.9 (3.7) days. The mean (SD) age of the mothers was $27.6(6.0)$ years. The major indications for admission for these neonates were; infections 300 (24.8\%), low birth weight (LBW) 307(25.4\%) and asphyxia 130(10.7). Out of these 1211 neonates, 985 $(81.3 \%)$ were discharged having improved; 133 (11\%) died and the remaining 93 (7.7\%), were discharged against medical advice. Preterm birth, LBW and birth asphyxia were the major causes of death among these neonates, the table.

\section{Discussion:}

Perhaps this is the first published data concerning nursery issue in Sudan. The study showed that infections, preterm birth, LBW and asphyxia were the main cause of admission. There were $11 \%$ neonatal deaths. Preterm birth, LBW and birth asphyxia were the major causes of death among these neonates. In Nigeria, neonatal mortality rates of $16.7 \%$ were reported, largely contributed by out-born infants ${ }^{10}$. However, these studies should be compared cautiously, because in our study only in-born neonates were investigated, while in Nigeria the majority of these deaths were out-born neonates.

In the current study, the majority of the admissions were due to LBW and preterm birth, which were the leading cause of neonatal deaths. LBW and preterm birth are usually intermingled and may be difficult to dissect especially in poor settings or when the data were retrospectively retrieved. Recently, we observed high prevalence of LBW and preterm birth from different regions of Sudan $^{5-8}$. Furthermore, we observed that, LBW neonates were at seven times higher risk of perinatal mortality and low APGAR score $^{5,}$. Preterm birth, infections and asphyxia are thought to be the main causes of death of newborn babies worldwide ${ }^{1}$. However, this is hospital based study and may not present what is going on in the community. Furthermore, the study investigated in-born babies only, women who are at risk of negative outcomes (e.g. referred cases) might be over presented, while community based studies may be less reliable with respect to accurate diagnosis of causes of death.

\section{Conclusion:}

Neonatal infection, preterm birth and LBW were the common causes of neonatal morbidity and mortality. There is an urgent need for more research concerning these common causes of morbidity and mortality.

\section{References}

1. Lawn JE, Cousens S, Zupan J. Lancet Neonatal Survival Steering Team. 4 million neonatal deaths: when? Where? Why? Lancet. 2005; 365(9462):891900.

2. United Nations. Resolution adopted by the General Assembly. S-27/2. A world fit for children. New York: United Nations; 2002. 
3. Haggaz AD, Radi EA, Adam I. High perinatal mortality in Darfur, Sudan. J Matern Fetal Neonatal Med. 2008; 21:277.

4. Haggaz AA, Radi EA, Adam I. High maternal mortality in Darfur, Sudan. Int J Gynaecol Obstet. 2007; 98: 252-3.

5. Adam I, Babiker S, Mohmmed AA et al. Low body mass index, anaemia and poor perinatal outcome in a rural hospital in eastern Sudan. J Trop Pediatr. 2008; 54:202-4.

6. Hassan AA, Abubaker MS, Radi EA et al. Education, prenatal care, and poor perinatal outcome in Khartoum, Sudan. Int J Gynaecol Obstet. 2009; 105: 66-67.
7. Alhaj AM, Radi EA, Adam I. Epidemiology of preterm birth in Omdurman maternity hospital, Sudan. J Matern Fetal Neonatal Med. 2009; 29:1-4

8. Haggaz AD, Radi EA, Adam I. Anaemia and Low birth weight in Western Sudan

9. Vik T, Vatten L, Markestad T et al. Dubowitz assessment of gestational age and agreement with prenatal methods. Am J Perinatol. 1997 ;14: 369-73.

10. Mukhtar-Yola M, Iliyasu ZA. review of neonatal morbidity and mortality in Aminu Kano Teaching Hospital, northern Nigeria. Trop Doct. 2007 ;37(3):130-2. 